\title{
NOTE ON GROUPS WITH AND WITHOUT FULL BANACH MEAN VALUE
}

\author{
ERLING FøLNER
}

In this note I shall prove two theorems. Theorem 1 is a simple consequence of the Main Theorem in [4, p. 245]. It shows that there exists an upper mean value in any group with a full Banach mean value (see $[4$, p. 243]), which is quite analogous to the usual upper mean value in abelian groups and has similar properties. As to Theorem 2, let me remark that a group $G$ has a full Banach mean value except when there exists a function $H(x)$ which is $\geqq 1$ for all $x$ and has the form

$$
H(x)=h_{1}(x)-h_{1}\left(x a_{1}\right)+\ldots+h_{n}(x)-h_{n}\left(x a_{n}\right),
$$

where $h_{1}, \ldots, h_{n}$ are bounded functions on $G$ and $a_{1}, \ldots, a_{n}$ elements from $G$; see [2, Theorem 4, p. 14]. Theorem 2 contains a surprisingly stronger result.

Professor B. Sz.-Nagy has kindly called my attention to some overlapping between my papers [2] and [4] and an earlier paper by J. Dixmier [1]; in particular Theorem 4 of $[2$, p. 14] (with $L$ the space of all bounded functions) and part of the sufficiency statement in the Main Theorem of $[4$, p. 245] are contained in Dixmier's paper.

For every bounded function $f(x)$ on a group $G$ with full Banach mean value we put

$$
\bar{M}_{0} f=\inf _{\mathscr{A}} \sup _{x} \Sigma \alpha_{n} f\left(x a_{n}\right),
$$

where the infimum is to be taken over all $\mathscr{A}=\left\{\alpha_{1}, \ldots, \alpha_{N} ; a_{1}, \ldots, a_{N}\right\}$, $\alpha_{n}>0, \sum \alpha_{n}=1, a_{n} \in G$.

THEOREM 1. In a group $G$ with full Banach mean value, the expression $\bar{M}_{0} f$ has the properties

$$
\begin{aligned}
& \bar{M}_{0} f \leqq \sup _{x} f(x), \\
& \bar{M}_{0}\{\lambda f\}=\lambda \bar{M}_{0} f, \quad \lambda \geqq 0, \\
& \bar{M}_{0}\{f+g\} \leqq \bar{M}_{0} f+\bar{M}_{0} g, \\
& \bar{M}_{0}\{f(x)-f(x a)\}=0
\end{aligned}
$$

Received February 14, 1957. 
Every right-invariant Banach mean value $M f$ on the space of all bounded functions on $G[2$, p. 14] satisfies

$$
-\bar{M}_{0}(-f) \leqq M f \leqq \bar{M}_{0} f,
$$

and for any fixed $f$ the Banach mean value $M f$ can be chosen arbitrarily in the interval (5).

Proof. If $M f$ is a right-invariant Banach mean value, we have

$$
M f=M\left\{\sum \alpha_{n} f\left(x a_{n}\right)\right\} \leqq \sup _{x} \Sigma \alpha_{n} f\left(x a_{n}\right)
$$

and hence $M f \leqq \bar{M}_{0} f$. The other part of (5) follows by replacing $f$ by $-f$ in the inequality just obtained. The existence of a right-invariant Banach mean value $M f$ on the space of all bounded functions on $G$, which for an arbitrary fixed $f$ can be chosen arbitrarily in the interval (5), is a consequence of (1), (2), (3), (4), and the theorem of Banach stated in [3].

In order to prove (1), (2), (3), (4) we consider

$$
\bar{M} f=\inf _{H} \sup _{x}(f(x)+H(x)),
$$

where the infimum is taken over all $H$ of the form (0). It is finite and has all the properties stated for $\bar{M}_{0} f$ in (1), (2), (3), (4); see [2, pp. 14-15]. We shall prove that $\bar{M}_{0} f=\bar{M} f$. Since the function

$$
\sum \alpha_{n} f\left(x a_{n}\right)=f(x)+\sum \alpha_{n}\left(f\left(x a_{n}\right)-f(x)\right)
$$

has the form $f(x)+H(x)$, it follows from the definitions of $\bar{M}_{0} f$ and $\bar{M} f$ that $\bar{M} f \leqq \bar{M}_{0} f$.

In order to prove that $\bar{M}_{0} f \leqq \bar{M} f$, let

$$
H(x)=h_{1}(x)-h_{1}\left(x a_{1}\right)+\ldots+h_{n}(x)-h_{n}\left(x a_{n}\right)
$$

be chosen such that $f(x)+H(x) \leqq \bar{M} f+\varepsilon$. By means of the Main Theorem in [4, p. 245], we can find, to every $\eta>0$, a finite set $E$ of elements from $G$ such that

$$
N\left(E \cap E a_{i}\right)>(1-\eta) N(E), \quad i=1, \ldots, n,
$$

where $N($.$) denotes the number of elements in the set between the$ brackets. Then

$$
\begin{aligned}
\bar{M} f+\varepsilon & \geqq N(E)^{-1} \sum_{a \in E}(f(x a)+H(x a)) \\
& =N(E)^{-1} \sum_{a \in E} f(x a)+\sum_{i=1}^{n} N(E)^{-1} \sum_{a \in E}\left(h_{i}(x a)-h_{i}\left(x a a_{i}\right)\right) \\
& \geqq N(E)^{-1} \sum_{a \in E} f(x a)-2 \eta \sum_{i=1}^{n} \sup _{x}\left|h_{i}(x)\right|
\end{aligned}
$$


Choosing $\eta$ sufficiently small we get

$$
N(E)^{-1} \sum_{a \in E} f(x a) \leqq \bar{M} f+2 \varepsilon,
$$

and the inequality $\bar{M}_{0} f \leqq \bar{M} f$ follows. This completes the proof of Theorem 1.

REMark. Theorem 1 remains valid when the word "right-invariant" is replaced by "bi-invariant", $\bar{M}_{0} f$ by

$$
\bar{M}_{2} f=\inf \sup _{x} \sum \alpha_{n} f\left(b_{n} x a_{n}\right),
$$

and (4) by $\bar{M}_{2}\{f(x)-f(b x a)\}=0$.

This may be proved in a similar way as Theorem 1 .

Theorem 2. In a group $G$ without full Banach mean value every bounded function can be uniformly approximated by functions of the form (0).

More generally: If $L$ is a right-translation invariant linear space of bounded functions on $G$ which is closed with respect to the formation of maximum and minimum between two functions, contains the constants, and has no right-invariant Banach mean value, then every function from $L$ can be uniformly approximated by functions

$$
H(x)=h_{1}(x)-h_{1}\left(x a_{1}\right)+\ldots+h_{n}(x)-h_{n}\left(x a_{n}\right),
$$

where the h's belong to $L$.

Proof. For a function $f$ from $L$ we put

$$
\bar{M} f=\inf ^{*} \sup _{x}(f(x)+H(x)),
$$

where the infimum is to be taken over all functions $H$ of the form (0) with $h$ 's belonging to $L$ and such that $f(x)+H(x) \geqq 0$. We remark that since $L$ has no right-invariant Banach mean value, there exists an $H_{0}(x)$ of the form (0) with $h$ 's from $L$ such that $\inf _{x} H_{0}(x)=1$ (see $[2$, p. 14]), and consequently the function

$$
H(x)=\left|\inf _{x} f(x)\right| H_{0}(x)
$$

has the form (0) with $h$ 's in $L$ and satisfies

$$
\begin{aligned}
\inf _{x}(f(x)+H(x)) & \geqq \inf _{x} f(x)+\inf _{x} H(x) \\
& =\inf _{x} f(x)+\left|\inf _{x} f(x)\right| \geqq 0 .
\end{aligned}
$$

Thus the set of $H$ 's over which the infimum in (6) is to be taken, is not empty. Further we obtain the inequality 
so that

$$
0 \leqq \bar{M} f \leqq \sup _{x} f(x)+\left|\inf _{x} f(x)\right| \sup _{x} H_{0}(x)
$$

$$
0 \leqq \bar{M} f \leqq C \sup _{x}|f(x)| \quad \text { with } \quad C=1+\sup _{x} H_{0}(x) \quad(\geqq 2) .
$$

We first show that

$$
\bar{M}(f+g) \leqq \bar{M} f+\bar{M} g .
$$

Let $\varepsilon>0$ be given. We choose $H_{1}(x)$ and $H_{2}(x)$ of the form (0) with $h$ 's in $L$ such that

$$
0 \leqq f(x)+H_{1}(x) \leqq \bar{M} f+\varepsilon \quad \text { and } \quad 0 \leqq g(x)+H_{2}(x) \leqq \bar{M} g+\varepsilon .
$$

Then

$$
0 \leqq f(x)+g(x)+H_{1}(x)+H_{2}(x) \leqq \bar{M} f+\bar{M} g+2 \varepsilon .
$$

Since $H_{1}+H_{2}$ is an $H$ with $h$ 's in $L$, we get

and (8) follows.

$$
\bar{M}(f+g) \leqq \bar{M} f+\bar{M} g+2 \varepsilon,
$$

Next we show that

$$
\bar{M}\{f(x a)\}=\bar{M}\{f(x)\} .
$$

We have

$$
\begin{aligned}
\bar{M}\{f(x)\} & =\inf ^{*} \sup _{x}(f(x)+H(x)) \\
& =\inf ^{*} \sup _{x}(f(x a)+\{f(x)-f(x a)+H(x)\}) \\
& =\bar{M}\{f(x a)\},
\end{aligned}
$$

where the infimum is to be taken over all $H$ 's of the form (0) with $h$ 's in $L$ and $f(x)+H(x) \geqq 0$.

Next we show that

$$
\bar{M}\{\lambda f(x)\}=\lambda \bar{M}\{f(x)\}, \quad \lambda \geqq 0 .
$$

In the case $\lambda=0$ we get

$$
\bar{M}\{0 f\}=\inf ^{*} \sup _{x} H(x)=0=0 \bar{M} f,
$$

where the infimum is to be taken over all $H$ 's of the form (0) with $h$ 's in $L$ and $H(x) \geqq 0$. We have used that $H(x)=0$ is admitted. In the case $\lambda>0$ we have

$$
\bar{M}\{\lambda f\}=\inf ^{*} \sup _{x}(\lambda f(x)+\lambda H(x)),
$$

where we consider $H$ 's with $h$ 's in $L$ and $\lambda f(x)+\lambda H(x) \geqq 0$. Thus (10) is clear also in this case.

Next we show that

$$
\bar{M}\{f(x)-f(x a)\}=0 .
$$


On the one hand, from (8) and (9) we get

$$
\bar{M}\{f(x)-f(x a)\} \geqq \bar{M} f(x)-\bar{M} f(x a)=0,
$$

on the other hand, from (6) we get

$$
\bar{M}\{f(x)-f(x a)\} \leqq \sup _{x}\{f(x)-f(x a)+f(x a)-f(x)\}=0
$$

since $0 \geqq 0$. Hence (11) follows.

We want to show that $\bar{M} f=0$ for all $f$ in $L$. We assume, to the contrary, that there exists an $f_{0}$ in $L$ with $\bar{M} f_{0} \neq 0$ (incidentally $\bar{M} f_{0}>0$ on account of (7)). By (8), (10), and the theorem of Banach stated in [3] we can determine a linear functional $M f$ on $L$ with $M f \leqq \bar{M} f$ for all $f$ in $L$ and $M f_{0}=\bar{M} f_{0}>0$. It follows from (11) that $M f$ is right-invariant. In a well-known manner we proceed as follows in order to write $M f$ as the difference between two positive linear functionals $M^{+} f$ and $-M^{-} f$.

For functions $f \geqq 0$ in $L$ we put

$$
M^{+} f=\sup _{\substack{0 \leqq g \leqq f, g \in L}} M g .
$$

From $M 0=0$ and (7) applied to $g$ we obtain the estimate

$$
0 \leqq M^{+} f \leqq C \sup _{: x} f(x) .
$$

Further $M^{+}\{\lambda f\}=\lambda M^{+} f$ when $\lambda \geqq 0$, and $M^{+}\{f(x a)\}=M^{+}\{f(x)\}$. For functions $f \geqq 0$ and $g \geqq 0$ in $L$ it is clear that

$$
M^{+}(f+g) \geqq M^{+} f+M^{+} g .
$$

In order to prove the converse inequality, let $h$ be in $L$ and let $0 \leqq h \leqq f+g$. It suffices to show that $h=f_{1}+g_{1}$ where $f_{1}$ and $g_{1}$ are in $L$ and satisfy the inequalities $0 \leqq f_{1} \leqq f$ and $0 \leqq g_{1} \leqq g$. Clearly, $f_{1}=\min (h, f)$ and $g_{1}=h-f_{1}$ have the desired properties.

Every function $f$ in $L$ can be written $f=f_{1}-f_{2}$ with non-negative $f_{1}$ and $f_{2}$ in $L$, in particular $f=f^{+}-f^{-}$, where

The equation

$$
f^{+}=\max (f, 0), \quad-f^{-}=\min (f, 0) .
$$

$$
M^{+} f=M^{+} f_{1}-M^{+} f_{2}
$$

defines $M^{+} f$ in a unique manner, and for arbitrary $f$ and $g$ in $L$ we get

$$
M^{+}\{\lambda f\}=\lambda M^{+} f
$$

(where $\lambda$ is arbitrary), $M^{+}(f+g)=M^{+} f+M^{+} g$, and $M^{+}\{f(x a)\}=M^{+}\{f(x)\}$. Further

$$
M^{+} f=M^{+} f^{+}-M^{+} f^{-} \leqq M^{+} f^{+} \leqq C \sup _{x} f^{+}(x) .
$$


This estimate, however, is not sufficient for our purpose. Using that the constants belong to $L$ we get instead

$$
M^{+} f \leqq M^{+}\left(\sup _{x} f(x)\right)=M^{+}(1) \sup _{x} f(x) .
$$

For functions $f \geqq 0$ in $L$ we put

It follows from (7) that

$$
M^{-} f=\inf _{\substack{0 \leqq g \leqq f, g \in L}} M g .
$$

so that

$$
M g \geqq-\bar{M}(-g) \geqq-C \sup _{x}|-g(x)| \geqq-C \sup _{x} f(x)
$$

$$
0 \geqq M^{-} f \geqq-C \sup _{x} f(x) .
$$

Continuing as above we define $M^{-} f$ for all $f$ in $L$ and show that $-M^{-} f$ has the same properties as those listed for $M^{+} f$.

We shall prove that

$$
M f=M^{+} f+M^{-} f .
$$

It suffices to do it for functions $f \geqq 0$ in $L$. Let $\varepsilon>0$ be given. We choose $g$ in $L$ such that $0 \leqq g \leqq f$ and

$$
M g>M^{+} f-\varepsilon .
$$

Then $0 \leqq f-g \leqq f$ so that $M(f-g) \geqq M^{-} f$. Hence

$$
M f>M^{+} f+M^{-} f-\varepsilon .
$$

Analogously we get

$$
M f<M^{+} f+M^{-} f+\varepsilon .
$$

Thus (12) is proved.

Since $M^{+} f_{0}+M^{-} f_{0}=M f_{0}>0$, either $M^{+} f_{0}$ or $M^{-} f_{0}$ is $\neq 0$. Assume, for instance, that $M^{+} f_{0}<0$. Then $M^{+} \mathbf{I} \neq 0$ (and hence $>0$ ) on account of the inequality

$$
M^{+}\left(-f_{0}\right) \leqq M^{+}(1) \sup _{x}\left(-f_{0}(x)\right),
$$

and $M^{+} f / M^{+} l$ is a right-invariant Banach mean value on $L$. Thus we have arrived at a contradiction.

We have shown that $\bar{M} f=0$ for all $f$ in $L$. Thus to every $\varepsilon>0$ there exists an $H$ with $h$ 's in $L$ such that

$$
\varepsilon \geqq f(x)+H(x) \geqq 0 .
$$

In other words: $f(x)$ can be uniformly approximated by functions $H(x)$ with $h$ 's in $L$. This completes the proof of Theorem 2 .

REMARK. Theorem 2 remains valid when "right-" is replaced by "bi-" and 


$$
h_{1}(x)-h_{1}\left(x a_{1}\right)+\ldots+h_{n}(x)-h_{n}\left(x a_{n}\right)
$$

by

$$
h_{1}(x)-h_{1}\left(b_{1} x a_{1}\right)+\ldots+h_{n}(x)-h_{n}\left(b_{n} x a_{n}\right) \text {. }
$$

This may be proved in a similar way as Theorem 2 .

\section{REFERENCES}

1. J. Dixmier, Les moyennes invariantes dans les semi-groupes et leurs applications, Acta Sci. Math. Szeged 12, Leopoldo Fejér et Frederico Riesz LXX annos natis dedicatus, Pars A (1950), 213-227.

2. E. Følner, Generalization of a theorem of Bogolioùboff to topological abelian groups. With an appendix on Banach mean values in non-abelian groups, Math. Scand. 2 (1954), 5-18.

3. E. Følner, Note on a generalization of a theorem of Bogolioùboff, Math. Scand. 2 (1954), 224-226.

4. E. Følner, On groups with full Banach mean value, Math. Scand. 3 (1955), 243-254.

THE TECHNICAL UNIVERSTTY OF DENMARK, COPENHAGEN 